ORIGINAL ARTICLE

\title{
KLF8 promotes human breast cancer cell invasion and metastasis by transcriptional activation of MMP9
}

\author{
X Wang ${ }^{1,4}, \mathrm{H} \mathrm{Lu}^{1}, \mathrm{AM}$ Urvalek$^{1,5}, \mathrm{~T} \mathrm{Li}^{1}, \mathrm{~L} \mathrm{Yu}^{1}, \mathrm{~J}_{\text {Lamar }^{2,6}, \mathrm{CM} \text { DiPersio }^{2} \text {, PJ Feustel }}^{3}$ \\ and $\mathbf{J} \mathrm{Zhao}^{1}$
}

${ }^{1}$ Burnett School of Biomedical Sciences, College of Medicine, University of Central Florida, Orlando, FL, USA; ${ }^{2}$ Center for Cell Biology and Cancer Research, Albany Medical College, Albany, NY, USA and ${ }^{3}$ Center for Neuropharmacology and Neuroscience, Albany Medical College, Albany, NY, USA

Epithelial to mesenchymal transition (EMT) and extracellular matrix degradation are critical for the initiation and progression of tumor invasion. We have recently identified Krüppel-like factor 8 (KLF8) as a critical inducer of EMT and invasion. KLF8 induces EMT primarily by repressing E-cadherin transcription. However, how KLF8 promotes invasion is unknown. Here, we report a novel KLF8-to- matrix metalloproteinase (MMP)9 signaling that promotes human breast cancer invasion. To identify the potential KLF8 regulation of MMPs in breast cancer, we established two inducible cell lines that allow either KLF8 overexpression in MCF-10A or knockdown in MDA-MB-231 cells. KLF8 overexpression induced a strong increase in MMP9 expression and activity as determined by quantitative real-time PCR and zymography. This induction was well correlated with the MMP inhibitor-sensitive Matrigel invasion. Conversely, KLF8 knockdown caused the opposite changes that could be partially prevented by MMP9 overexpression. Promoter-reporter assays and chromatin and oligonucleotide precipitations determined that KLF8 directly bound and activated the human MMP9 gene promoter. Threedimensional (3D) glandular culture showed that KLF8 expression disrupted the normal acinus formation, which could be prevented by the MMP inhibitor, whereas KLF8 knockdown corrected the abnormal 3D architecture, which could be protected by MMP9 overexpression. KLF8 knockdown promoted MDA-MB-231 cell aggregation in suspension culture, which could be prevented by MMP9 overexpression. KLF8 knockdown inhibited the lung metastasis of MDAMB-231 cells in nude mice. Immunohistochemical staining strongly correlated the co-expression of KLF8 and MMP9 with the patient tumor invasion, metastasis and poor survival.

Correspondence: Dr J Zhao, Burnett School of Biomedical Sciences, College of Medicine, University of Central Florida, Orlando, FL 32827, USA.

E-mail: jizhao@mail.ucf.edu

${ }^{4}$ Current address: Ge*NY*sis Center for Excellence in Cancer Genomics, University at Albany, Rensselaer, NY 12144, USA.

${ }^{5}$ Current address: Department of Pharmacology, Weill Medical College of Cornell University, New York, NY 10065, USA.

${ }^{6}$ Current address: Howard Hughes Medical Institute, David H Koch Institute for Integrative Cancer Research, Massachusetts Institute of Technology, Cambridge, MA 02139, USA.

Received 22 May 2010; revised 11 October 2010; accepted 25 October 2010; published online 13 December 2010
Taken together, this work identified the KLF8 activation of MMP9 as a novel and critical signaling mechanism underlying human breast cancer invasion and metastasis.

Oncogene (2011) 30, 1901-1911; doi:10.1038/onc.2010.563; published online 13 December 2010

Keywords: KLF8; MMP9; invasion; metastasis; human mammary epithelial cells; human breast cancer

\section{Introduction}

Breast cancer survival rate falls from $90 \%$ for localized to $20 \%$ for metastatic disease. Metastasis requires invasive cells to detach from the localized tumors by epithelial to mesenchymal transition (EMT), degrade the extracellular matrix using proteases including matrix metalloproteinases (MMPs), survive the circulation as circulating tumor cells (CTCs) and colonize at distant locations. Understanding of the molecular mechanisms behind each of the steps is essential for targeting metastatic cells at early stage to improve patient survival.

Krüppel-like factor 8 (KLF8) is a GT-box (CACCC) binding dual-transcription factor that has a critical role in the regulation of cell cycle progression (Zhao et al., 2003; Wei et al., 2006; Wang et al., 2008; Mehta et al., 2009; Urvalek et al., 2010), transformation (Wang and Zhao, 2007), EMT and invasion (Wang et al., 2007). $\mathrm{KLF} 8$ recruits the $\mathrm{CtBP}$ co-repressor (van Vliet et al., 2000) to repress or the p300 and p300/CBP associated factor (PCAF) co-activators to activate target gene promoters (van Vliet et al., 2000; Zhao et al., 2003; Zhang et al., 2005; Wei et al., 2006; Wang et al., 2007; Evans and Liu, 2010; Lloyd, 2010; Urvalek et al., 2010). KLF8 expression is promoted by Src and phosphatidylinositol 3 kinase signaling (Zhao et al., 2003; Ding et al., 2005; Wang et al., 2008) and its transcription is activated by Sp1 (Wang et al., 2008) and KLF1 (Eaton et al., 2008) or inhibited by KLF3 (Eaton et al., 2008). KLF8 is also regulated by posttranslational sumoylation (Wei et al., 2006) and localization (Mehta et al., 2009; Rodriguez and Martignetti, 2009). We previously identified KLF8 as a focal adhesion kinase (FAK) downstream effector (Zhao et al., 2003). FAK is a critical 

invasion and metastasis (Zhao and Guan, 2009). Like FAK, KLF8 is highly overexpressed in invasive human cancers including breast cancer (Wang and Zhao, 2007) and promotes breast cancer cell invasion (Wang et al., 2007). However, the mechanisms by which KLF8 promotes human breast cancer invasion have not been investigated.

In this study, we report that KLF8 regulates MMP9 expression by transcriptional activation in human breast cancer cells to promote cell invasion and metastasis in mice. We also show a strong correlation of KLF8 and MMP9 co-expression with the patient tumor metastasis. Our results support a potentially important role for KLF8 in promoting human breast cancer metastasis.

\section{Results}

\section{KLF8 upregulates MMP9 expression and activity to promote cell invasion}

Degradation of extracellular matrix and vascular basement membrane is required for a cancer cell to invade. MMP9 and MMP2 are among the major proteinases that have such a role. Interestingly, during the invasion of fibroblasts and other cell types, the expression and activity of these MMPs are found to be upregulated by FAK (Schlaepfer and Mitra, 2004), the upstream regulator of KLF8.

We first tested whether KLF8 is sufficient to promote invasion using our tet-off NIH3T3/KLF8 cells (Zhao et al., 2003). We found that induction of KLF8 expression led to a greater than threefold increase in the cell invasiveness (Figure 1A, left panel, compare induced (I) with uninduced (U)). We then determined whether KLF8 regulates the MMPs by in-gel zymography. We found that induction of KLF8 expression resulted in an increase in both pro-MMP9 and active MMP9, and only active MMP2 in the cells (Figure 1A, right-top panel, compare I with $\mathrm{U}$ ). This result suggests that KLF8 regulate MMP9 mainly at the transcriptional level whereas KLF8 activation of MMP2 may be through an indirect mechanism. We have demonstrated that KLF8 promotes the MCF-10A cell invasiveness (Wang et al., 2007). To test whether KLF8 also regulates the MMPs in these cells, we performed in-gel zymography using MCF-10A, the non-invasive (MCF-7) and the highly invasive (MDA-MB-231) human breast cancer cell lines (Figure 1A, right-bottom panel). In agreement with the literature (Yao et al., 2001; Tsai et al., 2003; Rahman et al., 2006), there was little or no expression of MMP9 or MMP2 in MCF-10A-Mock cells (lane 1) (Wang et al., 2007) and MCF-7 (lane 3), but a dramatic increase in the MCF-10A-KLF8 (Wang et al., 2007) (compare lanes 2 to 1) and MDA-MB-231 cells (compare lanes 4 to 3 ).

We then established a MCF-10A cell line that expresses inducible KLF8 (10A-iK8) for an in-depth study. The inducible expression was verified by western blotting (Figure 1B, left panel, top two rows, compare lanes I with $\mathrm{U}$ ). To determine KLF8 regulation of the
MMPs at the message levels, we performed reverse transcriptase-PCR and quantitative real-time PCR and found that KLF8 increased mRNA expression of MMP9 but not MMP2 (Figure 1B, compare lanes or columns $\mathrm{U}$ with I). This is consistent with the change in pro-forms of the MMPs shown in Figure 1A.

To confirm the role of KLF8 activation of MMPs for the cell invasion, we performed in situ zymography using both the 10A-iK8 cells and MG6001 (Figure 1C). We found that KLF8 expression caused the matrix degradation (compare I + DMSO with $\mathrm{U}+\mathrm{DMSO}$ ), which is MG6001-sensitive (compare I+ GM6001 with I + DMSO) and consistent with the cell invasiveness (Figure 1D, compare columns 3 to 2 and 1).

Taken together, these results suggest that KLF8 activation of MMPs is critical for its promotion of cell invasion.

\section{$M M P 9$ is a direct transcriptional activation target of $K L F 8$}

To test whether KLF8 regulates MMP9 transcription, we performed prompter (MMP9p) reporter (Lamar et al., 2008) assays. We found that KLF8 activated MMP9p in both NIH3T3 and MCF-10A cells (Figure 2a, compare the black with white columns). However, the activation domain deficient mutant (mKLF8) (Urvalek et al., 2010) was no longer able to do so (Figure 2a, compare the gray with black and white columns). These results suggest that KLF8 regulates MMP9p by transcriptional activation.

To test whether KLF8 directly activates MMP9p, we first analyzed the $1.6 \mathrm{~kb}$ promoter region and found six GT-boxes (Figure 2b). To map the promoter region essential for KLF8-responsive GT-box(s), we generated MMP9p truncation mutants (Figure 2b). Although deletion of the $-1.6 \mathrm{~kb}$ through $-541-\mathrm{bp}$ region only caused a slight decrease in MMP9p activity (compare columns $\mathrm{d} 6, \mathrm{~d} 5-6$ or $\mathrm{d} 4-6$ with wild type), deletion to -359 bp position caused a $\sim 80 \%$ decrease (compare columns d3-6 with wild type). Further deletion did not have an effect (compare columns d2-6 or d1-6 with d3-6). This result suggests that the region between $-541 \mathrm{bp}$ and $-359 \mathrm{bp}$ is essential for the promoter activation by KLF8. Interestingly, this region contains a GT-box (GT-box 3).

We disrupted this GT-box to see if it mediates KLF8 activation of MMP9p in the $10 \mathrm{~A}-\mathrm{iK} 8$ cells. Unlike the wild-type promoter that responded well to KLF8 in a time- and thus KLF8 level-dependent manner (Figure 2c, black columns), the mutant promoter (mGT-box 3) completely lost the responsiveness to KLF8 (Figure 2c, white columns).

To test whether KLF8 interacts with the endogenous MMP9 promoter in the 10A-iK8 cells, we performed chromatin immunoprecipitation assays (Figure 2d, top panel). We found that the promoter fragment containing the GT-box 3 was specifically co-immunoprecipitated by anti-KLF8 but not the control immunoglobulin $\mathrm{G}$ from I (compare lanes 3 to 2 and 4 ) but not $\mathrm{U}$ cells (lane 3, compare $\mathrm{U}$ with $\mathrm{I}$ ). These results suggest that 

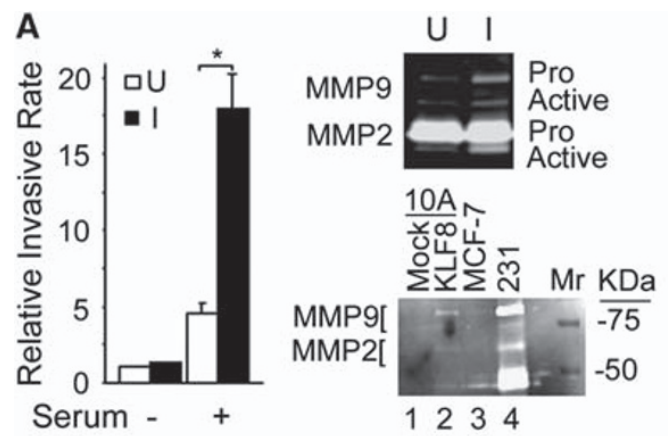

B
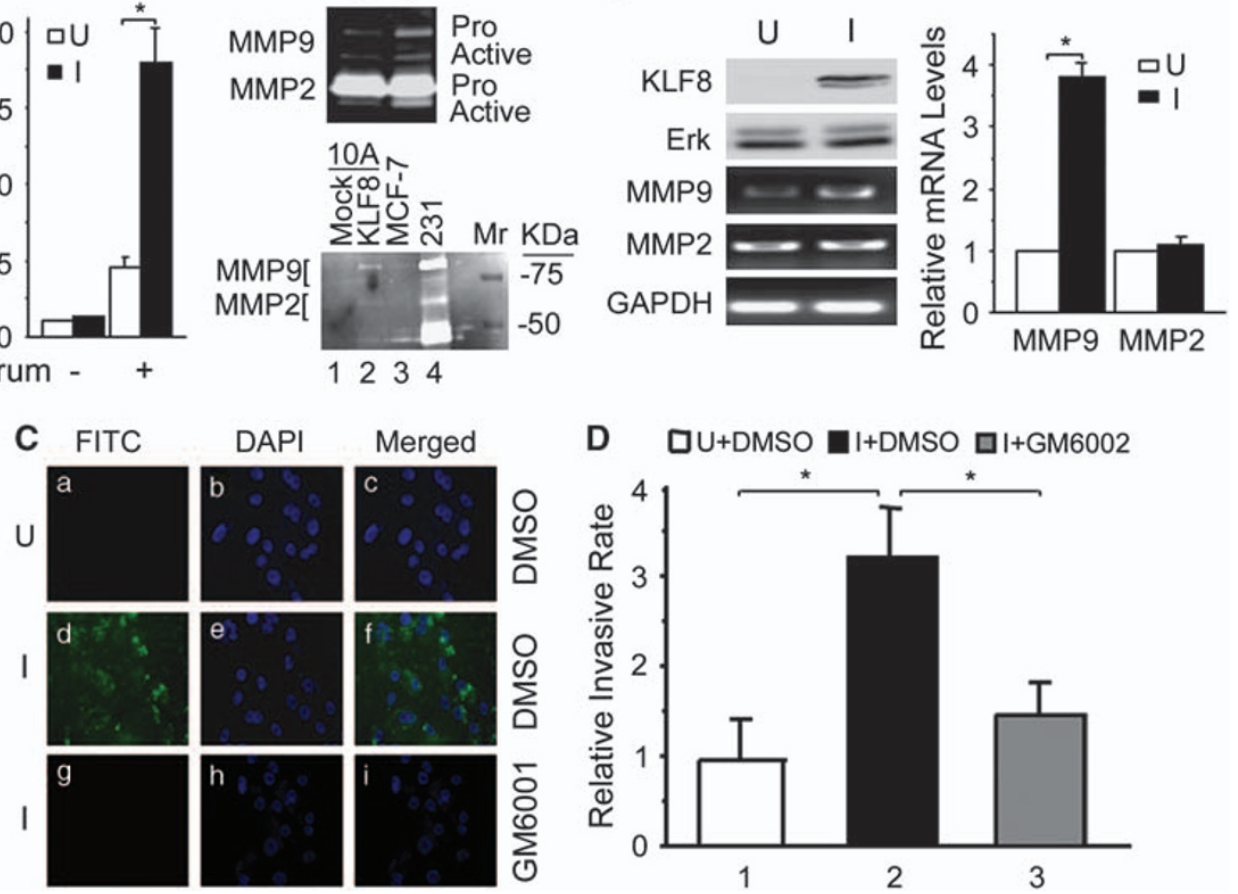

Figure 1 KLF8 upregulates the expression and activity of MMP9 and promotes cell invasion. (A) KLF8 promotes NIH3T3 cell invasion and upregulates MMP9 in both NIH3T3 and MCF-10A cells. Induced (I) and uninduced (U) Tet-off NIH3T3/KLF8 cells were examined for invasion through Matrigel (left panel). The MMP activities in the media from either the same 3T3/KLF8 cells (right-top panel) or the indicated normal or cancer cell lines (right-bottom panel) were examined by in-gel zymography after 3-5 days of incubation. (B) Induction of MMP9 mRNA expression by KLF8. The 10A-iK8 cells were grown under I or U conditions for $48 \mathrm{~h}$. Whole cell lysates or total RNA were prepared for western blotting with anti-HA using Erk as a loading control, or reverse transcriptase (RT)-PCR and quantitative real-time PCR (qRT-PCR) using glyceraldehyde 3-phosphate dehydrogenase (GAPDH) as an internal control (left panel). (C) KLF8 activates MMPs in situ. The 10A-iK8 cells were cultured under U (a-c) or I conditions with DMSO (d-i) or $20 \mu \mathrm{M}$ of GM6001 (g-i) in the medium and processed for in situ zymography. (D) KLF8-induced invasion is blocked by the MMP inhibitor. Matrigel invasion assays were conducted using the $10 \mathrm{~A}$-iK 8 cells described in $\mathbf{B}$. ${ }^{*} P<0.05$.

KLF8 could bind directly to the MMP9 promoter at the GT-box 3 site.

To see whether the KLF8-MMP9p interaction requires the GT-box 3, we performed biotinylated oligonucleotide precipitation assays (Figure 2d, bottom panel). We showed that KLF8 strongly bound to the wild-type (compare lanes 2 to 3) but not the GT-box 3 mutant (mGT) MMP9p fragment (compare lanes 1 to 2 or 3 ).

Taken together, these results suggest that KLF8 activates MMP9p by directly binding to it at the GT-box 3.

\section{Aberrant upregulation of MMP9 by KLF8 in human breast cancer cells is critical for the cell invasion} For an in-depth understanding of how KLF8 regulates breast cancer cell invasion and metastasis, we also established the 231-K8ikd cell line that expresses inducible KLF8 short hairpin RNA. Green fluorescent protein (GFP) labels all the cells. The induced knockdown of KLF8 was confirmed by red fluorescent protein (RFP) expression (Figure 3A, compare panels f with c), western blotting (Figure 3B, top panel, compare lanes I with $\mathrm{U}$ ) and quantitative real-time PCR (Figure 3B, middle and bottom panels, compare lanes I with U).
Using this cell line, we first tested whether KLF8 is required for the MMP mRNA expression. We found that the KLF8 knockdown caused a threefold decrease in MMP9 but had no effect on MMP2 mRNA levels (Figure 3B, middle and bottom panels, compare lanes I with U). In-gel zymography showed that on KLF8 knockdown, both the pro- and active MMP9 were dramatically reduced, whereas only active MMP2 but not the pro-MMP2 was decreased (Figure 3C, top panel, compare lanes I with U). MMP9p reporter assay indicated that the MMP9p activity was reduced by $60 \%$ when KLF8 was silenced (Figure 3C, bottom panel, compare lanes I with U).

Consistent with our previous observations using transient KLF8 knockdown (Wang et al., 2007), there was $>50 \%$ reduction in the cell invasiveness on the inducible KLF8 knockdown. Importantly, this was correlated with a decrease in MMP9 protein levels (Figure 3D, compare columns or lanes 2 to 1 ). To determine the requirement of MMP9 for cell invasion, we transiently silenced MMP9 and found that the cell invasiveness dropped by $40 \%$ (Figure 3D, compare lanes or columns 4 to 3). To test whether MMP9 can rescue the cell invasiveness during KLF8 knockdown, we overexpressed MMP9 in the cells and found that the 

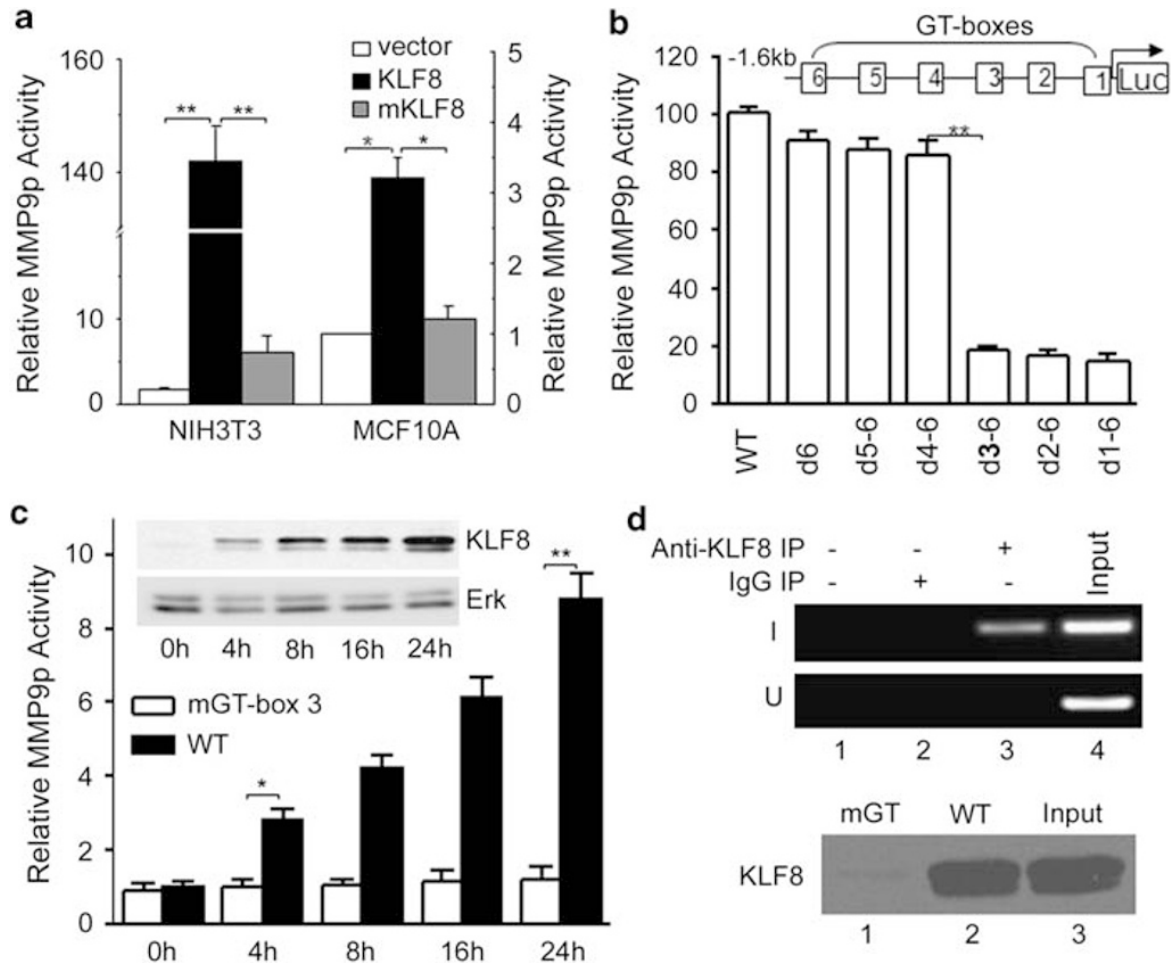

Figure 2 The GT-box located at the -449 bp of MMP9 promoter is the primary mediator of the activation of MMP9 transcription by KLF8. (a) KLF8 activates MMP9 promoter (MMP9p) in both NIH3T3 and MCF-10A cells. Reporter activity was performed as described in Materials and methods section. (b) The minimal KLF8-responding MMP9p region contains three GT-boxes. A serial MMP9p mutants (for example, d6) were co-transfected KLF8 and luciferase activities were measured after 16h. (c) The GT-box at $-449 \mathrm{bp}$ is crucial for MMP9p activation by KLF8. Uninduced 10A-iK8 cells were transfected with the $-1.6 \mathrm{~kb}$ MMP9p (WT) or its mutant (mGT-box 3, GGGTG to TGAGA mutation). After a $16 \mathrm{~h}$, the KLF8 expression was induced for indicated periods of time as confirmed by western blotting (inset) and luciferase assays were performed. (d) KLF8 directly binds to the endogenous MMP9p at the GT-box 3. The 10A-iK 8 cells were grown under U or I conditions for $72 \mathrm{~h}$ before chromatin immunoprecipitation (ChIP) (top panel) or biotinylated oligonucleotide precipitation (BOP) (bottom panel) assays as described in Materials and methods section. WT, wild-type and mGT, GT-box 3 mutated oligos. ${ }^{*} P<0.05 ;{ }^{* *} P<0.01$.

cell invasiveness was recovered regardless of KLF8 knockdown (Figure 3D, compare columns or lanes 6 to 5). These results suggest that the KLF8 knockdownmediated decrease in MMP9 mRNA levels was responsible for the decrease in MMP9 protein expression and activity, which in turn limits the cancer cell invasiveness.

In addition, we found that in three-dimensional (3D) spheroid culture the normal structure of acini (Debnath et al., 2003a; Lee et al., 2007) formed by the 10A-iK8 cells was disrupted on induction of KLF8 expression and treatment with GM6001 partially protected this disruption (Supplementary Figure S2A). Conversely, the aberrant 3D structures (Debnath et al., 2003b; Carrio et al., 2005; McLachlan et al., 2006; Park et al., 2006) formed by the 231-K8ikd cells was partially reversed by either treatment with GM6001 or induction of KLF8 knockdown (as indicated by the expression of RFP in the cells) and the latter was prevented by MMP9 ectopic expression (Supplementary Figure S2B).

Taken together, these results suggest that KLF8 activation of MMP9 transcription is essential for maintaining MMP9 expression and activity in the breast cancer cells and the cell invasiveness possibly by disrupting the normal and maintaining the abnormal glandular structure.
KLF8-MMP9 axis is critical for the cell disaggregation in suspension

It is implicated that single-cell migration is important for breast cancer cell invasion, circulation and metastasis (Giampieri et al., 2009) and single CTC circulation is also critical for extravasation (Whipple et al., 2008; Balzer et al., 2009).

To test whether KLF8 has a role in maintaining the non-aggregated status of CTC-mimicking suspended breast cancer cells, we analyzed the aggregation ability of the 231-K8ikd cells (Figure 4). We found that the cell aggregation was induced within $30 \mathrm{~min}$ in suspension culture under I but not $\mathrm{U}$ conditions (Figures $4 \mathrm{a}$ and $\mathrm{b}$, compare 5 to 2 or 6 to 4 ). This result was verified by an independent spheroid formation approach (Figures $4 \mathrm{c}$ and $\mathrm{d}$, compare I with $\mathrm{U}$ ). In this assay, the cells in which KLF8 was not knocked down (U, labeled by GFP only) grew sparsely. In contrast, when KLF8 was knocked (I, labeled by both GFP and RFP) the cells tended to aggregate together to form spheroids. To test whether MMP9 has a role in this regulation by KLF8, we performed rescue experiments and found that MMP9 overexpression prevented the spheroid formation regardless of KLF8 knockdown (Figures 4c and d, compare I + MMP9 and I). 
A
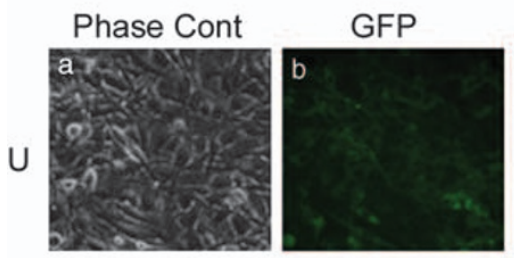

I

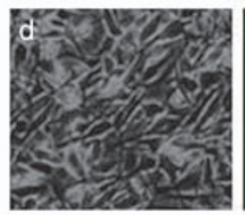

B
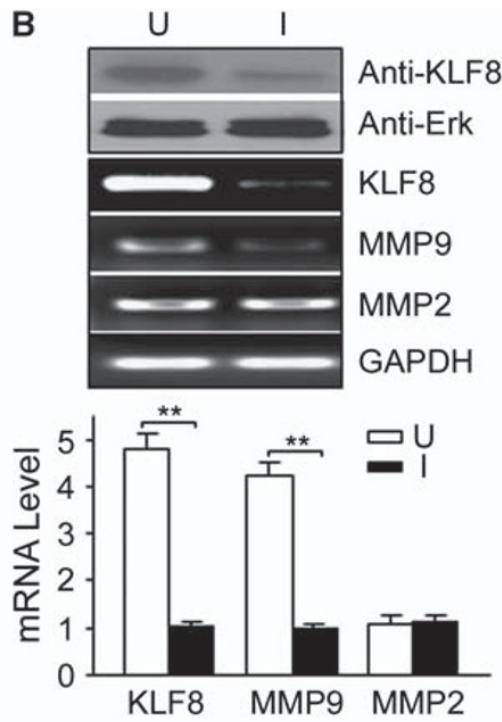

C
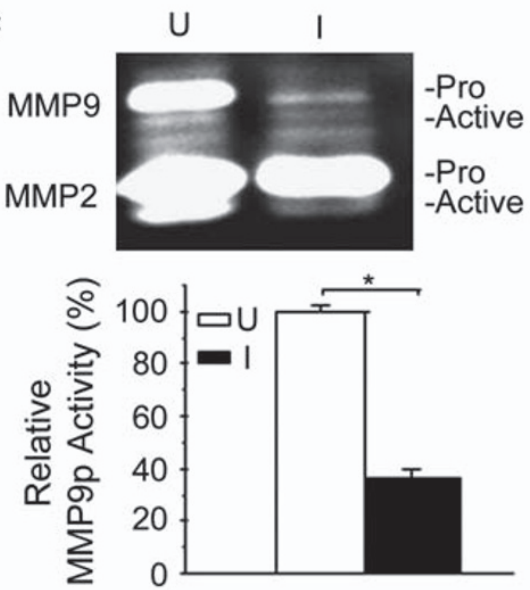

D

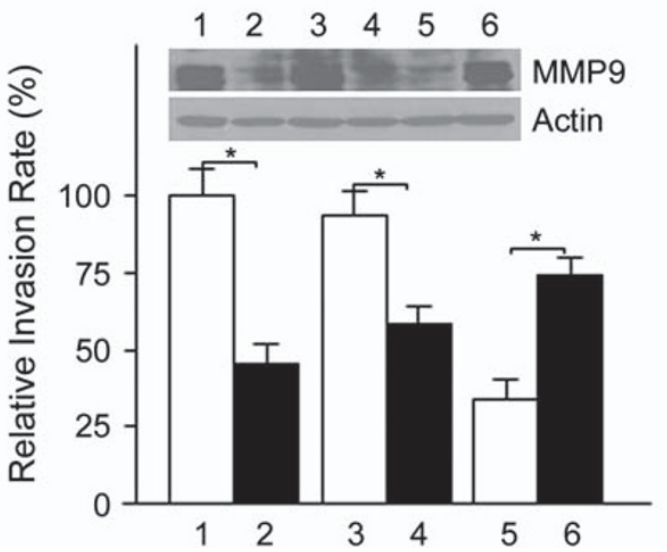

Figure 3 Knockdown of KLF8 causes a decrease in the expression and activity of MMP9 and cell invasion. (A) 4175TGL cells expressing inducible KLF8 short hairpin RNA (shRNA) (231-K8ikd). Images of the cells grown under U (a-c) and I (e, f) conditions. GFP labels all the cells whereas RFP represents those that express KLF8 shRNA. (B) KLF8 knockdown blocks the expression of MMP9 mRNA. The 231-K8ikd cells were grown under the indicated conditions for $72 \mathrm{~h}$. Western blotting and RNA analysis were conducted as in Figure 1B. (C), KLF8 knockdown inhibits enzymatic and promoter activities of MMP9. 231-K8ikd cells were grown for $72 \mathrm{~h}$ under the indicated conditions and processed for in-gel zymography (top panel) and promoter reporter assays (bottom panel) as in Figures 1a and 2b, respectively. (D) KLF8 knockdown-mediated inhibition of invasion can be prevented by overexpressing ectopic MMP9. The 231-K8ikd cells were maintained under the indicated conditions with or without MMP9 knockdown or overexpression for $72 \mathrm{~h}$ before Matrigel invasion assays. MMP9 protein was monitored by western blotting (inset). 1, U; 2, I; 3, $\mathrm{U}+$ control small interfering RNA (siRNA); 4, U + MMP9 siRNA; 5, I + Vector control; 6, I + MMP9 overexpression. $* P<0.05$; $* * P<0.01$.

These results suggest that the KLF8-MMP9 axis has an important role for single-cell invasion during breast cancer metastasis.

The aberrant overexpression of KLF8 is crucial for the lung metastasis

All the results described above pointed out a possibility that KLF8 expression is potentially important for the progression of tumor metastasis. To test this possibility, the 231-K8ikd cells were injected into nude mice through tail veins and the lung metastasis of the cells was examined (Figure 5). Knockdown of KLF8 resulted in approximately $75 \%$ reduction in the metastasis (Figure 5, compare I with U).
This result clearly demonstrates the critical role of KLF8 in the regulation of the breast cancer metastasis.

\section{Aberrant co-overexpression of KLF8 and MMP9 \\ is correlated with patient tumor metastasis}

The above results support a possibility that KLF8 could promote MMP9 expression in human breast tumors and thus tumor invasion and metastasis. To test this, we examined the co-expression of KLF8 and MMP9 in human breast tumor tissues by immunohistochemistry using two tissue arrays from different sources. One of the arrays consists of 15 duplicates of breast tumor specimens with matched normal tissue counterparts (IMH-371, Figures 6A and B and Supplementary 
a
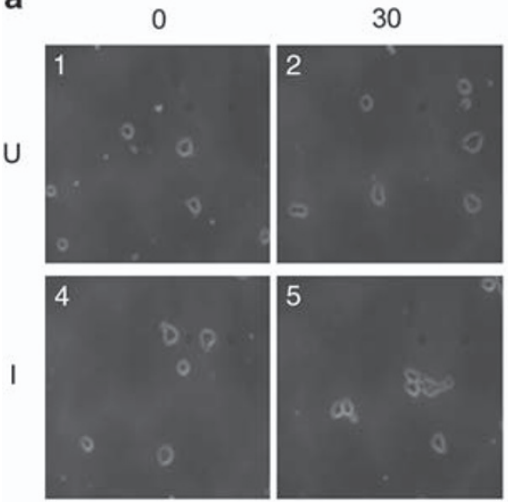

30

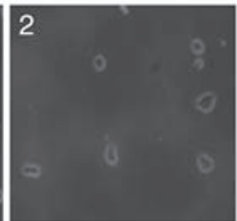

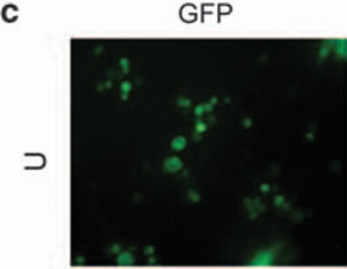
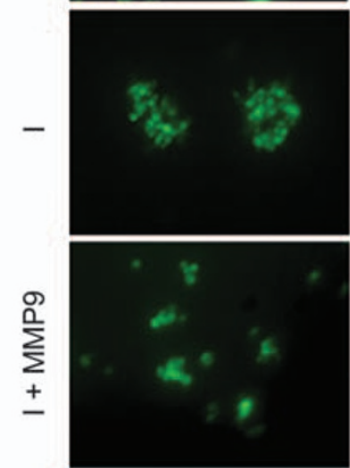
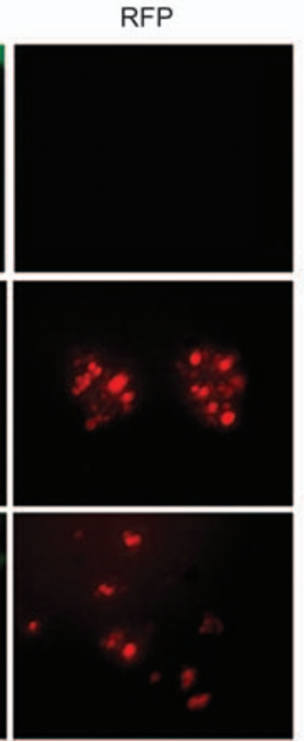

$60 \mathrm{~min}$
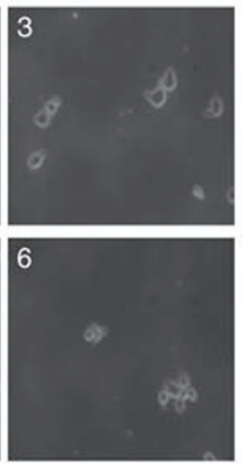

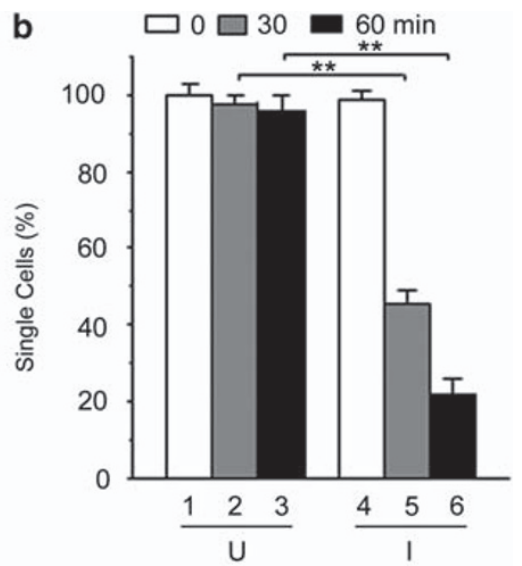

d

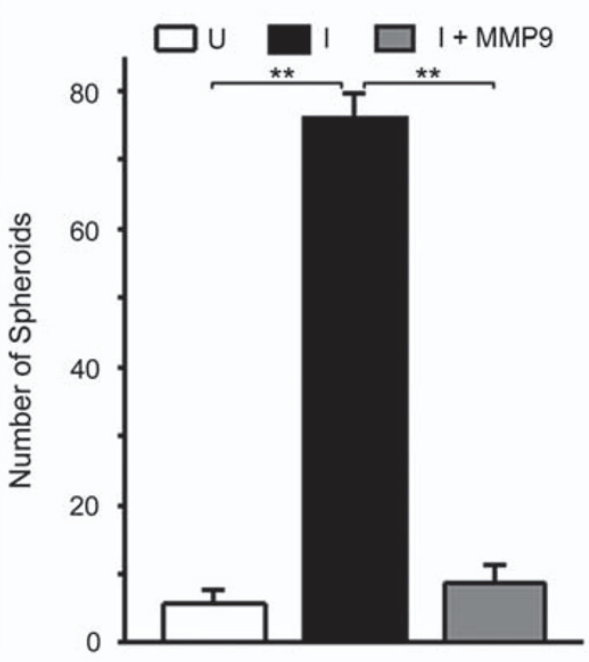

Figure 4 KLF8 activation of MMP9 is required for maintaining the non-aggregation status of breast cancer cells in suspension. (a, b) KLF8 knockdown promotes cell aggregation. The 231-K8ikd cells were grown under U or I conditions on a dish for 3 days. The cells were trypsinized, washed, re-suspended in whole medium and incubated with rotation. At the indicated time points, $50 \mu l$ of cells were carefully transferred to a hemocytometer for microscopic imaging (a) and single-cell counting (b). KLF8 knockdown was monitored by fluorescent microscopy of RFP expression as shown in c. (c, d) KLF8 knockdown promotes spheroid formation which can be prevented by overexpressing ectopic MMP9. The 231-K8ikd cells were grown as in a and $\mathbf{b}$ with or without ectopic expression of MMP9. Equal numbers of the cells in $50 \mu \mathrm{l}$ were used for 'hanging drop' culture as described in the Materials and methods section. After $24 \mathrm{~h}$, the fluorescent images were taken where GFP labeled all the cells and RFP displays only those with KLF8 knockdown (c) and spheroids containing at least 30 cells were counted (d). Data represent the mean \pm s.d. for at least three independent experiments. ${ }^{* *} P<0.01$.

Table B). The other is composed of 75 specimens representing normal through aggressive breast cancer tumors (BR1503, Figure 6C and Supplementary Table C). As expected, KLF8 was predominantly localized in the nuclei whereas the MMP9 showed both cytoplasmic and extracellular presence (Figure 6A and Supplementary Figure S3). Among all specimens of the small group examined, $47 \%$ were positive for both KLF8 and MMP9 (Supplementary Table B). Importantly, the KLF8 + /MMP9 + tumors were associated with a significantly higher metastasis rate (Figure 6B) and poor patient survival (Supplementary Table B). Similarly, among all the large set of 75 samples of normal tissue, benign tumor and malignant cyctosarcoma phyllodes, intraductal and invasive ductal carcinoma (Supplementary Table C), there was a statistically significant association of expression of KLF8 with MMP9 expression $(P<0.001)$ (Figure 6C1). KLF8 and/or MMP9 expression was present almost exclusively in invasive ductal carcinoma in which 35 of 60 invasive ductal samples showed one or both compared with 1 of 15 samples of tissue other than invasive ductal carcinoma (in this one case it was MMP9 expression alone). Thus, there was a statistically significant association of KLF8, MMP9 or both expression with the invasive potential $(P<0.001)$ (Figure 6C3). Indeed, in the 60 invasive ductal carcinoma samples only, there were 25 samples showing expression of both KLF8 and MMP9 and their expression was again statistically significantly associated with each other $(P<0.001)$ (Figure 6C2).

Collectively, these results strongly suggest that KLF8 activation of MMP9 transcription and expression has a 
a

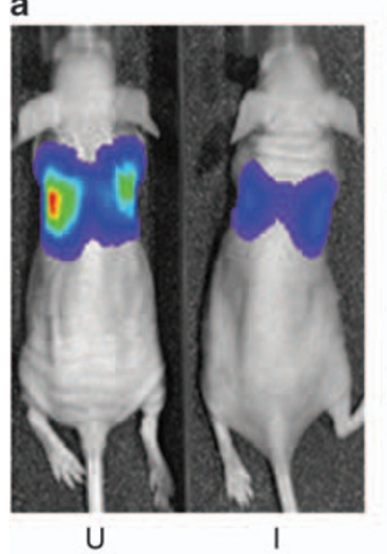

b

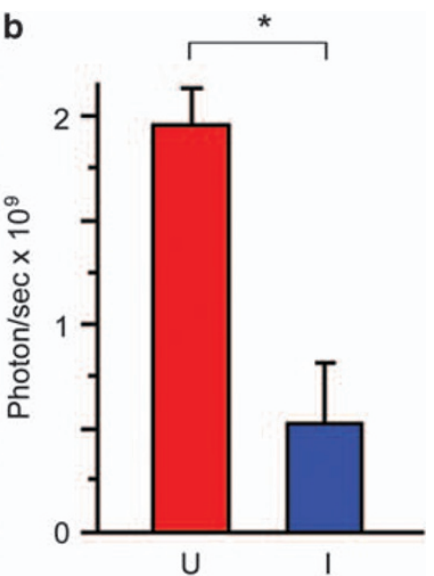

Figure 5 KLF8 knockdown suppresses lung metastasis of breast cancer. 4175TGL-KLF8ikd cells were injected through the tail vein. Mice were fed with food containing (I, KLF8 knockdown induced) or without doxycycline (U, KLF8 knockdown uninduced) for 6 weeks. Mice were anaesthetized and injected with D-luciferin followed by bioluminescence imaging analysis of the lung metastasis as described in Materials and methods section. (a) Representative images of the lung metastasis. (b) Quantitative results for the volumes of the lung metastasis. ${ }^{*} P<0.01$.

critical part in promoting human breast cancer invasion and metastasis.

\section{Discussion}

In this report, we identified the KLF8 regulation of MMP9 as a novel mechanism for human breast cancer cell invasion and metastasis. First, we showed that KLF8 promotes MMP9 transcription and activity in human breast cancer cells. Second, we demonstrated that the MMP activity is required for KLF8-induced cell invasion, the invasive 3D glandular architecture and the loss of cell-cell aggregation capability in suspension. Third, we provided evidence that KLF8 is required for breast cancer lung metastasis in model mice. Fourth, we identified a strong correlation of the co-expression of KLF8 and MMP9 with the patient tumor metastasis. This work has revealed a potentially significant role for KLF8 in promoting human breast cancer metastasis and shed a new light on the underlying molecular mechanisms.

This study identified MMP9 as a novel target of transcriptional activation by KLF8. This was demonstrated by (1) that KLF8 directly binds in vivo to the MMP9 promoter at the GT box and (2) both the KLF8binding site and activation domain are required for its activation of the MMP9 promoter (see Figure 2). Notably, both this GT-box and the KLF8 activation domain are well conserved across species (see Supplementary Figure) (Urvalek et al., 2010), suggesting a broad significance of this important KLF8 regulation of MMP9. Our finding that KLF8 activates MMP9 transcription is also supported by our gene expression profiling studies using the $10 \mathrm{~A}-\mathrm{iK} 8$ cells (data not shown). It is known that MMP9 message stability is also aberrantly regulated in invasive breast cancer (Iyer

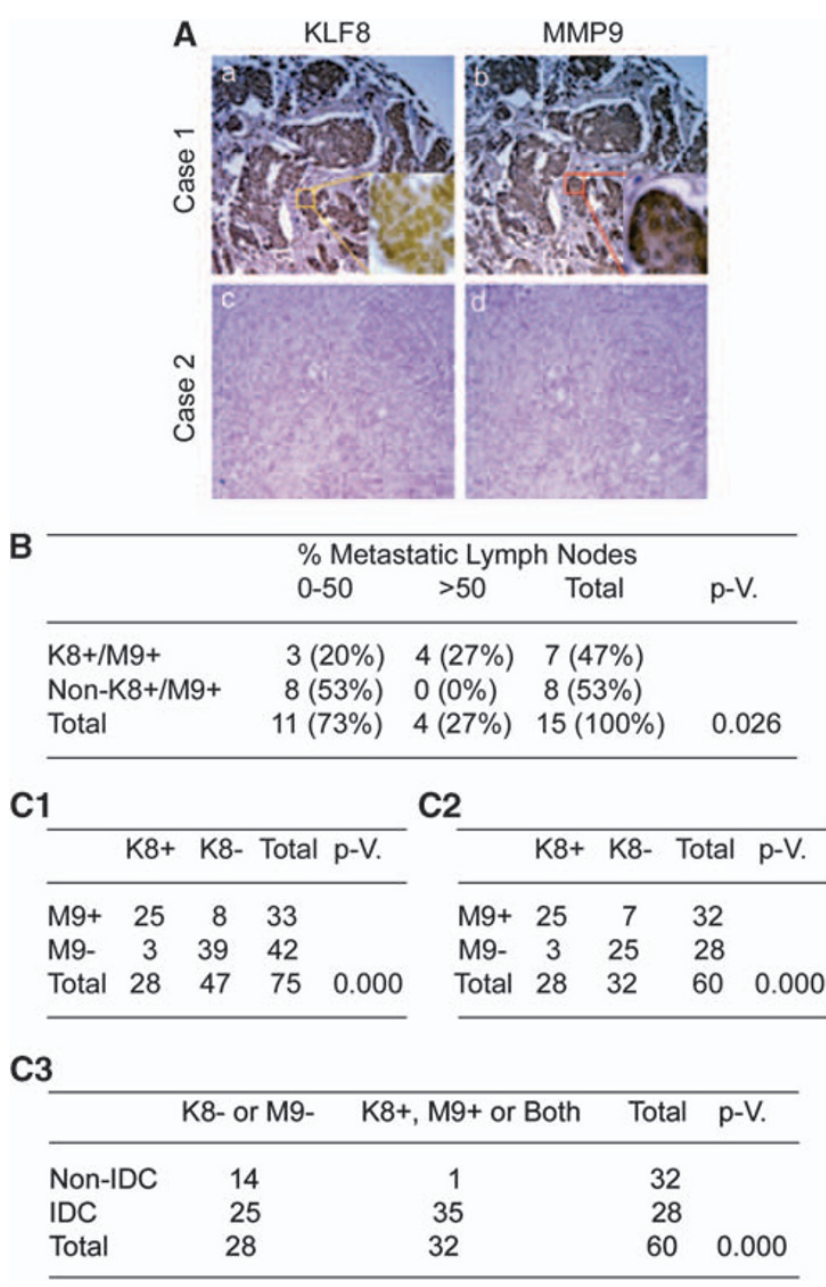

Figure 6 Positive correlation between KLF8 and MMP9 expression in metastatic breast cancer patient tumors. (A) MMP9 positive and negative surgical specimens of human breast tumors (IMH371) were subject to immunohistochemistry (IHC) co-staining as described in Materials and methods section for the expression of KLF8 (a, c) and MMP9 (b, d). The data represent 15 duplicates of breast tumor specimens with matched normal tissue counterparts (see Supplementary Table B) case 1 and case 2 represent positive and negative staining, respectively, for both KLF8 and MMP9. The zoom-ins of the indicated areas are shown in the insets. (B) Summary of the correlation. The correlation between the aberrant KLF8/MMP9 co-overexpression and the percentage of metastatic lymph nodes was analyzed by Fisher's exact test. K8, KLF8; M9, MMP9; $P$-v, $P$-value. (C) Similar data obtained from another set of 75 human tumor specimens (BR1503) (See Supplementary Table C). Four more cases positive for both KLF8 and MMP9 were shown in Supplementary Figure S3. Association of expression of KLF8 with MMP9 expression in all the specimens (C1), or of KLF8 expression with MMP9 expression in invasive ductal carcinoma of only (C2), or of either KLF8, MMP9 or both expression with invasive intraductal carcinoma (C3) was analyzed by $\chi^{2}$-test. A $P$-value of $<0.05$ was set as the criterion for statistical significance.

et al., 2005; Yan and Boyd, 2007; Lamar et al., 2008). The message stability is controlled primarily by its $3^{\prime}$ UTR. Our MMP9 promoter reporter, however, (see Figure 1) does not contain the $3^{\prime}$-UTR-encoding template and there is no evidence that KLF8 might regulate $3^{\prime}$-UTR of any gene. Therefore, it is unlikely that KLF8 also has a role in stabilizing MMP9 message. 
We have shown that KLF8 inhibits the E-cadherin transcription during EMT (Wang et al., 2007). We noticed that this EMT induction took several days although the E-cadherin mRNA began to decrease much earlier (data not shown). This observation suggests that the early repression of E-cadherin transcription by KLF8 is not sufficient to induce the EMT and destruction of the pre-existing E-cadherin protein is also essential. The shedding product of E-cadherin ectodomain, that is, the soluble E-cadherin, has been found in the blood and urine of metastatic cancer patients (Cristofanilli, 2006). Recent studies have demonstrated that the shedding is done by MMP9, MT1-MMP or MMP2 in lung or skin cancer cells during EMT (Covington et al., 2006; Dwivedi et al., 2006; Symowicz et al., 2007). On the other hand, treatment with the soluble E-cadherin has been shown to in turn upregulate the MMPs (Nawrocki-Raby et al., 2003) and promote the cell invasion (Symowicz et al., 2007). Additionally, overexpression of full-length E-cadherin can reduce MT1-MMP expression and MMP2 activity in the cells (Ara et al., 2000; Nawrocki-Raby et al., 2003). Our expression profiling data showed that MMP9 and MT1-MMP are upregulated within $24 \mathrm{~h}$ of KLF8 induction in the 10A-iK8 cells (data not shown). These results support an interesting possibility that during the early stage of KLF8-induced EMT, the pre-existing E-cadherin protein is downregulated by this shedding mechanism and the KLF8-activated MMPs may have a role for this potentially novel mechanism for the initiation of invasion at the early stage of breast cancer metastasis. Alternatively, KLF8 could use proteolytic degradation (Janda et al., 2006; Maeda et al., 2006; Yang et al., 2006) to destroy the pre-existing E-cadherin proteins. Nevertheless, the potentially mutual regulation between E-cadherin and the MMPs may represent a novel way by which KLF8 promotes the initiation of human breast cancer invasion by regulating both target gene transcription and protein stability. Using our inducible expression cell lines, we are vigorously testing this interesting possibility.

Single CTC circulation and extravasation is critical for breast cancer metastasis (Giampieri et al., 2009). The microtubule-based microtentacles formed on the surface of single CTCs are essential for the cells to adhere to and penetrate through the blood vessel endothelial layer during breast cancer cell extravasation (Whipple et al., 2008). Importantly, microtentacle formation is closely associated with vimentin (Whipple et al., 2008), a hallmark of EMT. Consistently, the disaggregation of the $10 \mathrm{~A}-\mathrm{iK}$ cells was clearly induced and maintained in suspension by KLF8 (Figure 4). As KLF8 has been shown to strongly induce EMT (Wang et al., 2007) and the KLF8-induced disaggregation depends on MMP9 (Figure 4b), it is plausible to speculate that the KLF8to-MMP9 signaling may have an important role in the formation and maintenance of single breast cancer CTCs and microtentacles to promote distant metastasis.

The tumor cell disaggregation could lead to either difficult cell movement resulting in reduced metastasis or increased efficiency of colonization and thus in- creased metastasis. Our results have clearly demonstrated that KLF8 is critical for the lung metastasis (Figure 5). Therefore, it is likely that KLF8-promoted single-cell circulation helps the cell metastasis by enhancing the metastatic colonization.

We have previously demonstrated that KLF8 downregulation of E-cadherin is critical for breast cancer cell invasion (Wang et al., 2007). Here, we show that KLF8mediated upregulation of MMP9 is also critical. In addition, the aberrant elevation of KLF8 is highly correlated with both the loss of E-cadherin (Wang et al., 2007) and the overexpression of MMP9 (Figure 6). These results strongly suggest that the downregulation of E-cadherin and upregulation of the MMP(s) by KLF8 are two important signaling arms required for maximizing KLF8-promoted breast cancer metastasis. Experiments are in progress to test this important possibility.

In summary, we have identified MMP9 as a novel target for transcriptional activation by KLF8 and demonstrated that the KLF8 regulation of MMP9 is critical for human breast cancer cell invasion. We have also shown that KLF8 expression is important for human breast cancer cell metastasis. Our results suggest a potentially significant role for KLF8 in the metastatic progression of human breast cancer. To date, MMP inhibitors have been proven less effective than expected in clinical trials (Coussens et al., 2002). Unlike MMP9, which is expressed in normal human tissue, the expression of KLF8 is barely detectable in normal epithelial cells (Wang and Zhao, 2007; Wang et al., 2007). In addition to MMP9, KLF8 also targets E-cadherin. Therefore, KLF8 may represent a novel favorable intervention target against breast cancer metastasis.

\section{Materials and methods}

Cell culture and reagents

MCF-10A (Debnath et al., 2003a; Wang et al., 2007) and the tet-off NIH 3T3/KLF8 cells were previously described (Zhao et al., 2003). The lung-prone MDA-MB-231 variant 4175 expressing thymidine kinase-GFP-luciferase was a kind gift from Dr Joan Massagué (Kang et al., 2005; Minn et al., 2005). These cells were maintained in either Dulbecco's modified Eagle's medium (DMEM)/F-12 or DMEM with $10 \%$ fetal bovine serum. To construct pLVUT-tTR-KRAB-KLF8, we replaced GFP with HA-KLF8 in the vector (Szulc et al., 2006). To construct pLVCT-tTR-KRAB-shKLF8, we cloned the short hairpin RNA duplex (Supplementary Table) into pLVTHM downstream of $\mathrm{H} 1$ promoter and then transferred the H1-shKLF8 cassette into pLVCT-tTR-KRAB (Szulc et al., 2006) and replacing the GFP with mCherry (RFP). The human MMP9 expressing lentiviral vector and MMP9 promoter reporter were previously described (Iyer et al., 2005; Lamar et al., 2008). To generate the MCF-10A line that expresses inducible KLF8 (10A-iK8) and the MDA-MB-231 line that expresses inducible KLF8 short hairpin RNA (231-K8ikd), the pLVUT-tTR-KRAB-KLF8 or pLVCT-tTR-KRAB-shKLF8 lentiviruses were used to infect the MCF-10A or 4175 cells and positive cells were cloned. The cell lines were maintained under $\mathrm{U}$ (in the absence of doxycycline or Dox) or I (in the presence of Dox) conditions depending on the experimental requirement. 


\section{In-gel zymography}

The MMP activities were assayed as described (Jorda et al., 2005). Briefly, $5 \times 10^{5}$ cells in a six-well plate were cultured in serum-free medium for 16-24h, and the conditioned medium was separated on an sodium dodecyl sulfate-polyacrylamide gel electrophoresis gel containing $1 \mathrm{mg} / \mathrm{ml}$ gelatin. The gel was washed with buffer I (Tris- $\mathrm{HCl}[\mathrm{pH} 7.5]$ and $2.5 \%$ Triton $\mathrm{X}-100)$, incubated overnight in buffer II (150 mM NaCl, $5 \mathrm{~mm}$ $\mathrm{CaCl}_{2}, 50 \mathrm{~mm}$ Tris- $\left.\mathrm{HCl}[\mathrm{pH} 7.6]\right)$ at $37^{\circ} \mathrm{C}$ and stained with Coomassie blue. The clear bands indicate where MMPs degraded gelatin.

In situ zymography

The $10 \mathrm{~A}-\mathrm{iK} 8$ cells were grown for $72 \mathrm{~h}$ under $\mathrm{U}$ or I conditions, $10^{6}$ cells were washed with DMEM and replated on DQ collagen fluorescein conjugate (Molecular Probes of Invitrogen, Carlsbad, CA, USA) coated cover glass and cultured with serum-free media for $16 \mathrm{~h}$. The fluorescence release resulting from MMP cleavage of the matrix was visualized by microscopy. The MMP inhibitor GM6001 was included in some cultures.

\section{Quantitative real-time PCR and western blotting}

These assays were done essentially as previously described (Wang et al., 2007). See primer information in Supplementary Table. Anti-MMP9 (1:1000) was from Cell Signaling Technology, Inc. (Danvers, MA, USA).

\section{Promoter reporter assays, chromatin immunoprecipitation and biotinylated oligonucleotide precipitation}

These assays were performed essentially as previously described (Wang et al., 2007). For reporter assays, cells were transfected with reporter constructs with transient or induced expression of KLF8. For chromatin immunoprecipitation assays, the 10A-iK8 cells were cultured under U or I conditions for 3 days and processed for the analyses. For biotinylated oligonucleotide precipitation assays, the $10 \mathrm{~A}-\mathrm{iK} 8$ cells were cultured with Dox for 3 days and cell lysates were processed for analysis. See oligouncleotide information in Supplementary Table.

\section{$3 D$ culture}

3D cell culture was performed as previously described (Debnath et al., 2003a). The 10A-iK8 cells were propagated in DMEM/F-12 with fetal bovine serum and other supplements. Single cells in medium containing 5\% Matrigel were seeded at a density of $5 \times 10^{4}$ cells $/ \mathrm{cm}^{2}$ on a Matrigel-coated well. The 231-K8ikd cells in DMEM plus $10 \%$ fetal bovine serum were seeded at $2 \times 10^{4}$ cells $/ \mathrm{cm}^{2}$. GM6001 was included in some experiments. In other experiments, the cells were infected with lentiviruses for 2 days to overexpress MMP9 before transfer to $3 \mathrm{D}$ culture.

\section{Cell aggregation analysis}

Trypsin-singularized cells were incubated in suspension $\left(1 \times 10^{6}\right.$ cells $\left./ \mathrm{ml}\right)$ for various periods of time, a $50 \mu \mathrm{l}$ volume of cells were carefully transferred to a cover slide. For spheroid formation in hanging drops, $2 \times 10^{4}$ cells were incubated in a $50 \mu \mathrm{l}$ drop of complete medium hanging on the inner side of the lid of 12-well plate for $12 \mathrm{~h}$. After the incubation, the drop was pipetted five times with a $200-\mu 1$ standard tip and spread on a cover slide. Images of more than five random fields from at least three independent cultures were taken by phase contrast or fluorescent microscopy. Single cells or spheroids that contain at least 30 cells were counted.
Immunohistochemistry

Human breast cancer tissue arrays (IMH-371 from IMGENEX and BR1503 from US Biomax, Rockville, MD, USA) sections were baked for $1 \mathrm{~h}$ at $62{ }^{\circ} \mathrm{C}$ for rehydration and microwaved in $0.01 \mathrm{~m}$ sodium citrate for $5 \mathrm{~min}$ for antigen retrieval. After incubated in $3 \% \mathrm{H}_{2} \mathrm{O}_{2}$ for $6 \mathrm{~min}$, the sections were serum blocked for $30 \mathrm{~min}$, incubated overnight at $4{ }^{\circ} \mathrm{C}$ with first antibodies in phosphate-buffered saline and subsequently with biotin-labeled secondary antibodies for $30 \mathrm{~min}$, followed by a peroxidase-labeled avidin-biotin complex (Vector Laboratories, Burlingame, CA, USA) for $30 \mathrm{~min}$. The sections were developed in 3,3-diaminobenzidine tetrahydrochloride for $2 \mathrm{~min}$ and counterstained with hematoxylin for $4 \mathrm{~min}$. The stained sections were dehydrated, treated with xylene and mounted for microscopy. Positive staining was displayed in brown color.

\section{Matrigel invasion assays}

The tet-off 3 T3/KLF8, 10A-iK8 or 231-K8ikd cells grown under $\mathrm{U}$ or I conditions for $24-48 \mathrm{~h}$ were assayed as previously described (Wang et al., 2007). In some experiments, transient MMP9 overexpression, knockdown or inhibition with GM6001 was included. The invasion rates were normalized to the $\mathrm{U}$ cells.

\section{Bioluminescence imaging analysis of lung metastasis}

All animal work was done in accordance with a protocol approved by the Institutional Animal Care and Use Committee. Female Balb/c nude mice (Taconic) 4-6 weeks old were used for all xenografting studies. For lung metastasis formation, $2 \times 10^{6}$ viable cells were washed and harvested in $0.1 \mathrm{ml}$ phosphate-buffered saline and subsequently injected into the lateral tail vein. In all, 5 or 6 weeks after injection mice were anaesthetized using isoflurance and injected intraperitoneally with $150 \mathrm{mg} / \mathrm{kg}$ of D-luciferin $(15 \mathrm{mg} / \mathrm{ml}$ in phosphate-buffered saline). Imaging was completed between 2 and 5 min after injection with a Xenogen IVIS system coupled to Living Image acquisition and analysis software (Caliper Life Sciences, Hopkinton, MA, USA). For BLI plots, photon flux (photons $/ \mathrm{s} / \mathrm{cm}^{2}$ per steradian) was measured with a region of interest drawn around the bioluminescence signal encompassing the thorax of the mouse in a prone position. A background value was subtracted that was obtained from a luciferin-injected control mouse.

\section{Statistical analysis}

Summary data are presented as mean \pm s.d. with a minimum of three observations per group. Unpaired, paired or single sample Student's $t$-test with the Bonferroni correction for the multiple comparisons was applied as appropriate. The two by two table for human data were analyzed by Fisher's exact test. Significance was determined by the alpha level of 0.05 .

\section{Conflict of interest}

The authors declare no conflict of interest.

\section{Acknowledgements}

We appreciate Dr Joan Massague for kindly providing the 4175 cells and Dr Chunhong Yan and Qingfen Li and Beihai Jiang of Zhao lab for helpful comments. This work was supported by grants from NCI (CA132977) and Susan G Komen for Cure (KG090444 and KG080616) to JZ and a grant from NCI (CA129637) to CMD. XW is a Komen postdoctoral fellow. 


\section{References}

Ara T, Deyama Y, Yoshimura Y, Higashino F, Shindoh M, Matsumoto A et al. (2000). Membrane type 1-matrix metalloproteinase expression is regulated by E-cadherin through the suppression of mitogen-activated protein kinase cascade. Cancer Lett 157: $115-121$.

Balzer EM, Whipple RA, Cho EH, Matrone MA, Martin SS. (2009). Antimitotic chemotherapeutics promote adhesive responses in detached and circulating tumor cells. Breast Cancer Res Treat 121: $65-78$.

Carrio M, Arderiu G, Myers C, Boudreau NJ. (2005). Homeobox D10 induces phenotypic reversion of breast tumor cells in a threedimensional culture model. Cancer Res 65: 7177-7185.

Coussens LM, Fingleton B, Matrisian LM. (2002). Matrix metalloproteinase inhibitors and cancer: trials and tribulations. Science 295: 2387-2392.

Covington MD, Burghardt RC, Parrish AR. (2006). Ischemia-induced cleavage of cadherins in NRK cells requires MT1-MMP (MMP-14). Am J Physiol Renal Physiol 290: F43-F51.

Cristofanilli M. (2006). Circulating tumor cells, disease progression, and survival in metastatic breast cancer. Semin Oncol 33: S9-S14.

Debnath J, Muthuswamy SK, Brugge JS. (2003a). Morphogenesis and oncogenesis of MCF-10A mammary epithelial acini grown in threedimensional basement membrane cultures. Methods 30: 256-268.

Debnath J, Walker SJ, Brugge JS. (2003b). Akt activation disrupts mammary acinar architecture and enhances proliferation in an mTOR-dependent manner. J Cell Biol 163: 315-326.

Ding Q, Grammer JR, Nelson MA, Guan JL, Stewart Jr JE, Gladson CL. (2005). p27Kip1 and cyclin D1 are necessary for focal adhesion kinase regulation of cell cycle progression in glioblastoma cells propagated in vitro and in vivo in the scid mouse brain. $J$ Biol Chem 280: 6802-6815.

Dwivedi DJ, Pino G, Banh A, Nathu Z, Howchin D, Margetts P et al. (2006). Matrix metalloproteinase inhibitors suppress transforming growth factor-beta-induced subcapsular cataract formation. $\mathrm{Am} \mathrm{J}$ Pathol 168: 69-79.

Eaton SA, Funnell AP, Sue N, Nicholas H, Pearson RC, Crossley M. (2008). A network of Kruppel-like factors (Klfs). Klf8 is repressed by Klf3 and activated by Klf1 in vivo. J Biol Chem 283: 26937-26947.

Evans PM, Liu C. (2010). New insights into KLF8-mediated transactivation. Cell Cycle 9: 649-650.

Giampieri S, Manning C, Hooper S, Jones L, Hill CS, Sahai E. (2009). Localized and reversible TGFbeta signalling switches breast cancer cells from cohesive to single cell motility. Nat Cell Biol 11: $1287-1296$.

Iyer V, Pumiglia K, DiPersio CM. (2005). Alpha3betal integrin regulates MMP-9 mRNA stability in immortalized keratinocytes: a novel mechanism of integrin-mediated MMP gene expression. $J$ Cell Sci 118: 1185-1195.

Janda E, Nevolo M, Lehmann K, Downward J, Beug H, Grieco M. (2006). Raf plus TGFbeta-dependent EMT is initiated by endocytosis and lysosomal degradation of E-cadherin. Oncogene 25: 7117-7130.

Jorda M, Olmeda D, Vinyals A, Valero E, Cubillo E, Llorens A et al. (2005). Upregulation of MMP-9 in MDCK epithelial cell line in response to expression of the Snail transcription factor. $J$ Cell Sci 118: $3371-3385$.

Kang Y, He W, Tulley S, Gupta GP, Serganova I, Chen CR et al. (2005). Breast cancer bone metastasis mediated by the Smad tumor suppressor pathway. Proc Natl Acad Sci USA 102: 13909-13914.

Lamar JM, Pumiglia KM, DiPersio CM. (2008). An immortalizationdependent switch in integrin function up-regulates MMP-9 to enhance tumor cell invasion. Cancer Res 68: 7371-7379.

Lee GY, Kenny PA, Lee EH, Bissell MJ. (2007). Three-dimensional culture models of normal and malignant breast epithelial cells. Nat Methods 4: 359-365.

Lloyd JA. (2010). KLF8 sets the pace for the cell cycle through interactions with p300 and PCAF. Cell Cycle 9: 650-651.
Maeda M, Johnson E, Mandal SH, Lawson KR, Keim SA, Svoboda RA et al. (2006). Expression of inappropriate cadherins by epithelial tumor cells promotes endocytosis and degradation of E-cadherin via competition for p120(ctn). Oncogene 25: 4595-4604.

McLachlan E, Shao Q, Wang HL, Langlois S, Laird DW. (2006). Connexins act as tumor suppressors in three-dimensional mammary cell organoids by regulating differentiation and angiogenesis. Cancer Res 66: 9886-9894.

Mehta TS, Lu H, Wang X, Urvalek AM, Nguyen KH, Monzur F et al. (2009). A unique sequence in the $\mathrm{N}$-terminal regulatory region controls the nuclear localization of KLF8 by cooperating with the C-terminal zinc-fingers. Cell Res 19: 1098-1109.

Minn AJ, Kang Y, Serganova I, Gupta GP, Giri DD, Doubrovin M et al. (2005). Distinct organ-specific metastatic potential of individual breast cancer cells and primary tumors. $J$ Clin Invest 115: $44-55$

Nawrocki-Raby B, Gilles C, Polette M, Bruyneel E, Laronze JY, Bonnet $\mathrm{N}$ et al. (2003). Upregulation of MMPs by soluble E-cadherin in human lung tumor cells. Int J Cancer 105: 790-795.

Park CC, Zhang H, Pallavicini M, Gray JW, Baehner F, Park CJ et al. (2006). Betal integrin inhibitory antibody induces apoptosis of breast cancer cells, inhibits growth, and distinguishes malignant from normal phenotype in three dimensional cultures and in vivo. Cancer Res 66: 1526-1535.

Rahman KM, Sarkar FH, Banerjee S, Wang Z, Liao DJ, Hong X et al. (2006). Therapeutic intervention of experimental breast cancer bone metastasis by indole-3-carbinol in SCID-human mouse model. $\mathrm{Mol}$ Cancer Ther 5: 2747-2756.

Rodriguez E, Martignetti JA. (2009). The Kruppel traffic report cooperative signals direct KLF8 nuclear transport. Cell Res 19: 1041-1043.

Schlaepfer DD, Mitra SK. (2004). Multiple connections link FAK to cell motility and invasion. Curr Opin Genet Dev 14: 92-101.

Symowicz J, Adley BP, Gleason KJ, Johnson JJ, Ghosh S, Fishman DA et al. (2007). Engagement of collagen-binding integrins promotes matrix metalloproteinase-9-dependent E-cadherin ectodomain shedding in ovarian carcinoma cells. Cancer Res 67: 2030-2039.

Szulc J, Wiznerowicz M, Sauvain MO, Trono D, Aebischer P. (2006) A versatile tool for conditional gene expression and knockdown. Nat Methods 3: 109-116.

Tsai MS, Shamon-Taylor LA, Mehmi I, Tang CK, Lupu R. (2003). Blockage of heregulin expression inhibits tumorigenicity and metastasis of breast cancer. Oncogene 22: 761-768.

Urvalek AM, Wang X, Lu H, Zhao J. (2010). KLF8 recruits the p300 and PCAF co-activators to its amino terminal activation domain to activate transcription. Cell Cycle 9: 601-611.

van Vliet J, Turner J, Crossley M. (2000). Human Kruppel-like factor 8: a CACCC-box binding protein that associates with CtBP and represses transcription. Nucleic Acids Res 28: 1955-1962.

Wang X, Urvalek AM, Liu J, Zhao J. (2008). Activation of KLF8 transcription by focal adhesion kinase in human ovarian epithelial and cancer cells. J Biol Chem 283: 13934-13942.

Wang X, Zhao J. (2007). KLF8 transcription factor participates in oncogenic transformation. Oncogene 36: 456-461.

Wang X, Zheng M, Liu G, Xia W, McKeown-Longo PJ, Hung MC et al. (2007). Kruppel-like factor 8 induces epithelial to mesenchymal transition and epithelial cell invasion. Cancer Res 67: 7184-7193.

Wei H, Wang X, Gan B, Urvalek AM, Melkoumian ZK, Guan JL et al. (2006). Sumoylation delimits KLF8 transcriptional activity associated with the cell cycle regulation. $J$ Biol Chem 281: 16664-16671.

Whipple RA, Balzer EM, Cho EH, Matrone MA, Yoon JR, Martin SS. (2008). Vimentin filaments support extension of tubulin-based microtentacles in detached breast tumor cells. Cancer Res 68: $5678-5688$

Yan C, Boyd DD. (2007). Regulation of matrix metalloproteinase gene expression. J Cell Physiol 211: 19-26. 
Yang JY, Zong CS, Xia W, Wei Y, Ali-Seyed M, Li Z et al. (2006). MDM2 promotes cell motility and invasiveness by regulating E-cadherin degradation. Mol Cell Biol 26: 7269-7282.

Yao J, Xiong S, Klos K, Nguyen N, Grijalva R, Li P et al. (2001). Multiple signaling pathways involved in activation of matrix metalloproteinase-9 (MMP-9) by heregulin-beta1 in human breast cancer cells. Oncogene 20: 8066-8074.

Zhang P, Basu P, Redmond LC, Morris PE, Rupon JW, Ginder GD et al. (2005). A functional screen for Kruppel-like factors that regulate the human gamma-globin gene through the CACCC promoter element. Blood Cells Mol Dis 35: 227-235.
Zhao J, Bian ZC, Yee K, Chen BP, Chien S, Guan JL. (2003). Identification of transcription factor KLF8 as a downstream target of focal adhesion kinase in its regulation of cyclin D1 and cell cycle progression. Mol Cell 11: 1503-1515.

Zhao J, Guan JL. (2009). Signal transduction by focal adhesion kinase in cancer. Cancer Metastasis Rev 28: 35-49.

(c) This work is licensed under the Creative Commons Attribution-NonCommercial-No Derivative Works 3.0 Unported License. To view a copy of this license, visit http://creativecommons.org/licenses/by-nc-nd/3.0/

Supplementary Information accompanies the paper on the Oncogene website (http://www.nature.com/onc) 